\title{
NEW RECORDS OF MACROFUNGI FROM TRABZON PROVINCE (TURKEY)
}

\author{
KELES, A. \\ Mathematics Van Yüzüncü Yıl University, Education Faculty, Department of Science and \\ Mathematics, Van, Turkey \\ (e-mail: alikeles61@yahoo.com; phone: +90 4322251701 ) \\ (Received $17^{\text {th }}$ Sep 2018; accepted $2^{\text {nd }}$ Jan 2019)
}

\begin{abstract}
This study was based on three macromycete samples collected during field studies carried out to determine the macrofungal biodiversity of Of and Çamburnu districts of Trabzon province (Turkey). As a result of necessary investigations, Pluteus variabilicolor Babos (Pluteaceae), Coprinopsis urticicola (Berk. \& Broome) Redhead, Vilgalys \& Moncalvo (Psathyrellaceae) and Chroogomphus confusus Y.C. Li \& Zhu L. Yang (Gomphidiaceae) were recorded from Turkey for the first time. Brief descriptions and photographs related to macro- and micromorphologies of the species are provided together with their localities, geographical positions, collection date and Genbank number related to genetic sequences.

Keywords: biodiversity, Basidiomycota, Pluteus, Coprinopsis, Chroogomphus
\end{abstract}

\section{Introduction}

Due to its excellent flora and climate (Dündar et al., 2016), Turkey is assumed to be very rich in naturally growing macrofungi, and currently comprises about 2,500 macrofungi species more than $85 \%$ of which belong to the phylum Basidiomycota (Kaya and Uzun, 2018). The Basidiomycota is a large fungal division with over 30,000 species. Most of the macrofungi such as agarics, bracket fungi, puffballs, earth stars, stinkhorns, boletes, etc., are included in this division. A great majority of the basidiomycota are terrestrial with wind-dispersed spores. Most of them are saprotrophic and are involved in litter and wood decay, but there are also pathogens of trees such as the honey fungus, Armillaria. Common woodland mushrooms grow in a mutually symbiotic relationship with the roots of trees, forming ectotrophic mycorrhiza. As saprotrophs, basidiomycetes play a vital role in recycling nutrients but they also cause severe damage as agents of timber decay. Basidiocarps of many mushrooms are edible, and some are grown commercially for food. It is also well known that the basidiocarps of certain mushrooms are poisonous (Webster and Weber, 2007).

During routine field studies in Trabzon province, some macromycete samples were collected and identified as Pluteus variabilicolor Babos, Coprinopsis urticicola (Berk. \& Broome) Redhead, Vilgalys \& Moncalvo and Chroogomphus confusus Y.C. Li \& Zhu L. Yang. Tracing the current literature it is found that almost 2200 basidiomycete taxa have been reported from Turkey, 24, 18 and 2 of which belong to the genera Pluteus, Coprinopsis and Chroogomphus respectively (Sesli and Denchev, 2014; Kaya, 2015; Solak et al., 2015; Akata et al., 2016; All1 et al., 2017; Uzun et al., 2017). But the check-lists on mycobiota of Turkey and recently contributed data (Iş1k and Türkekul, 2017; Kaya et al., 2016; Keleş and Oruç, 2017; Kaya and Uzun, 2018; Sadullahoğlu and Demirel, 2018; Sesli, 2018, Uzun and Acar, 2018; Uzun and Kaya, 2018; Uzun et al., 2018) reveals Pluteus variabilicolor, Coprinopsis urticicola and Chroogomphus confusus have not been previously reported from Turkey. The study aims to make a contribution to the mycobiota of Turkey by adding new records. 


\section{Materials and methods}

\section{Macrofungi sampling}

Macrofungi samples were collected from Of and Çamburnu districts of Trabzon province in 2012 and 2014 (Figure 1). Trabzon province has surface area of $4664 \mathrm{~km} 2$ and located in eastern Black Sea Region of Turkey, where the annual average temperature is $14.7^{\circ} \mathrm{C}$ and the average precipitation is $819.6 \mathrm{~mm}$. During field studies, required ecological and morphological characteristic of the samples were noted and they were photographed in their natural habitats. Then the samples were taken to the fungarium. Microscopic investigations were carried out under a Leica DM500 light microscope. Morphological identification of the specimens were carried out with the help of Gierczyk et al. (2014), Li et al. (2009), Migliozzi (2011), Desjardin et al. (2015) and Breitenbach and Kränzlin (1991). Using molecular methods, ITS region was investigated to conform the morphological identification. The samples are kept at the fungarium of Van Yüzüncü Y1l University in Van (VANF).

\section{Molecular studies}

Total DNA was extracted from dry fungarium materials using a EurX GeneMATRIX Plant \& Fungi DNA isolation kit (Poland). The internal transcribed spacer (ITS) regions, including the 5.8S nrDNA were amplified by the polymerase chain reaction (PCR) with the primer pair ITS1-F/ITS4 (White et al., 1990; Gardes and Bruns, 1993). The primer sequences used are given below;

- ITS1-F 5' TCCGTAGGTGAACCTGCGG 3'

- ITS4 5' TCCTCCGCTTATTGATATGC 3'

The amplification conditions were set as follows: denaturation at $95^{\circ} \mathrm{C}$ for $5 \mathrm{~min}, 35$ cycles of $45 \mathrm{~s}$ at $95^{\circ} \mathrm{C}, 45 \mathrm{~s} \min$ at $52^{\circ} \mathrm{C}, 60 \mathrm{~s}$ at $72^{\circ} \mathrm{C}$, and a final extension of $5 \mathrm{~min}$ at $72^{\circ} \mathrm{C}$. The PCR products were purified using the ExoSAP-IT ${ }^{\mathrm{TM}}$ PCR Product Cleanup Reagent and sequenced with an ABI 3730XL DNA analyzer (Applied Biosystems, Foster City, CA) and an BigDye Terminator v3.1 Cycle terminator cycle sequencing kit (Applied Biosystems, Foster City, CA). Consensus sequences where assembled using in BioEdit software, CAP contig.

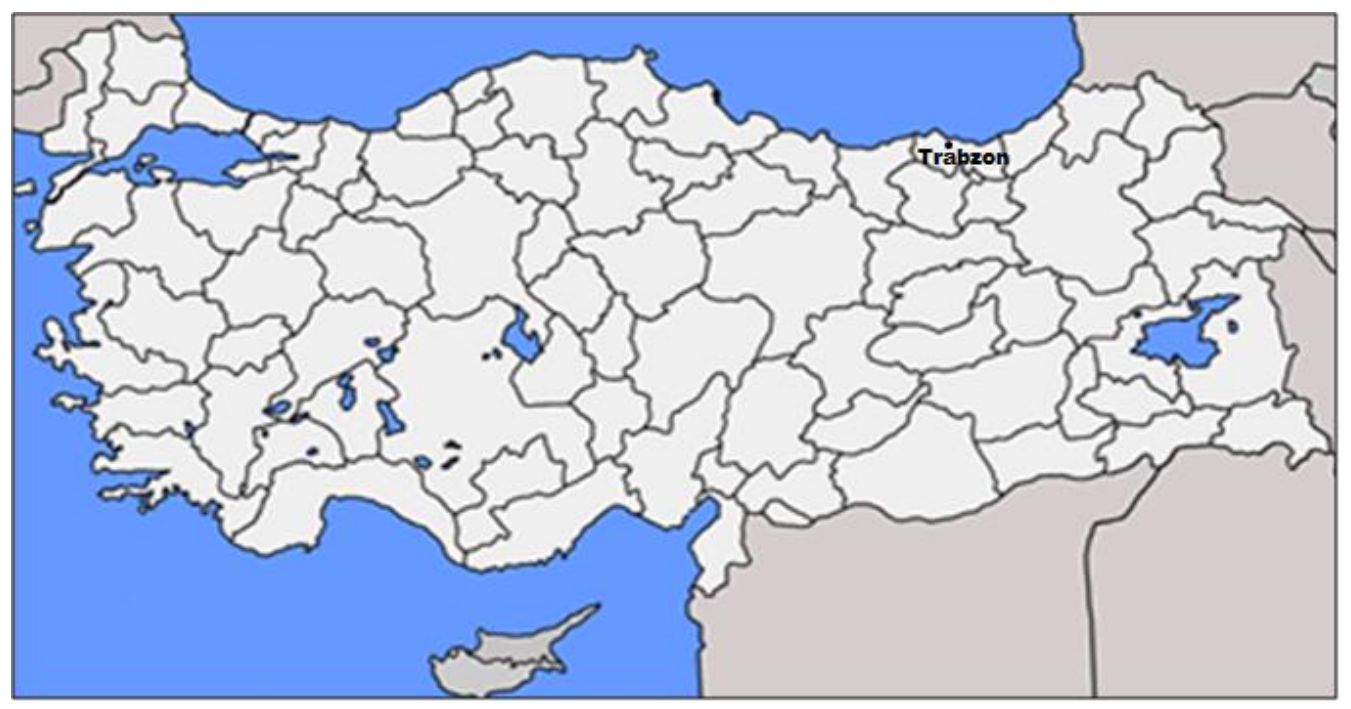

Figure 1. Species collection locations 


\section{Results}

Descriptions, photographs of fruiting bodies and images of microcharacters are provided. The taxonomy of the taxa are in accordance with Kirk et al. (2008).

\section{Pluteus variabilicolor Babos}

\section{Macroscopic and microscopic features}

Pileus 3-6 cm wide, surface smooth and streaked up to half, yellow and central umbo. Lamellae free, rather dense, creamy white. Stipe 2.5-6 $\times 0.4-0.7 \mathrm{~cm}$, surface smooth, cylindrical, slightly enlarged at the base, striatum length, whitish yellow. Basidia 20-28 × 5.8-8 $\mu \mathrm{m}$, clavate, 4-spores. Spores 5.4-7.0 $\times$ 4.5-5.6 $\mu \mathrm{m}$, ellipsoid, subglobose, thin-walled. Cheilocystidia 40-48 $\times 12-15 \mu \mathrm{m}$, hyaline, thin-walled, short. Pleurocystidia 65-120 × 19-35 $\mu \mathrm{m}$ (Figure 2).

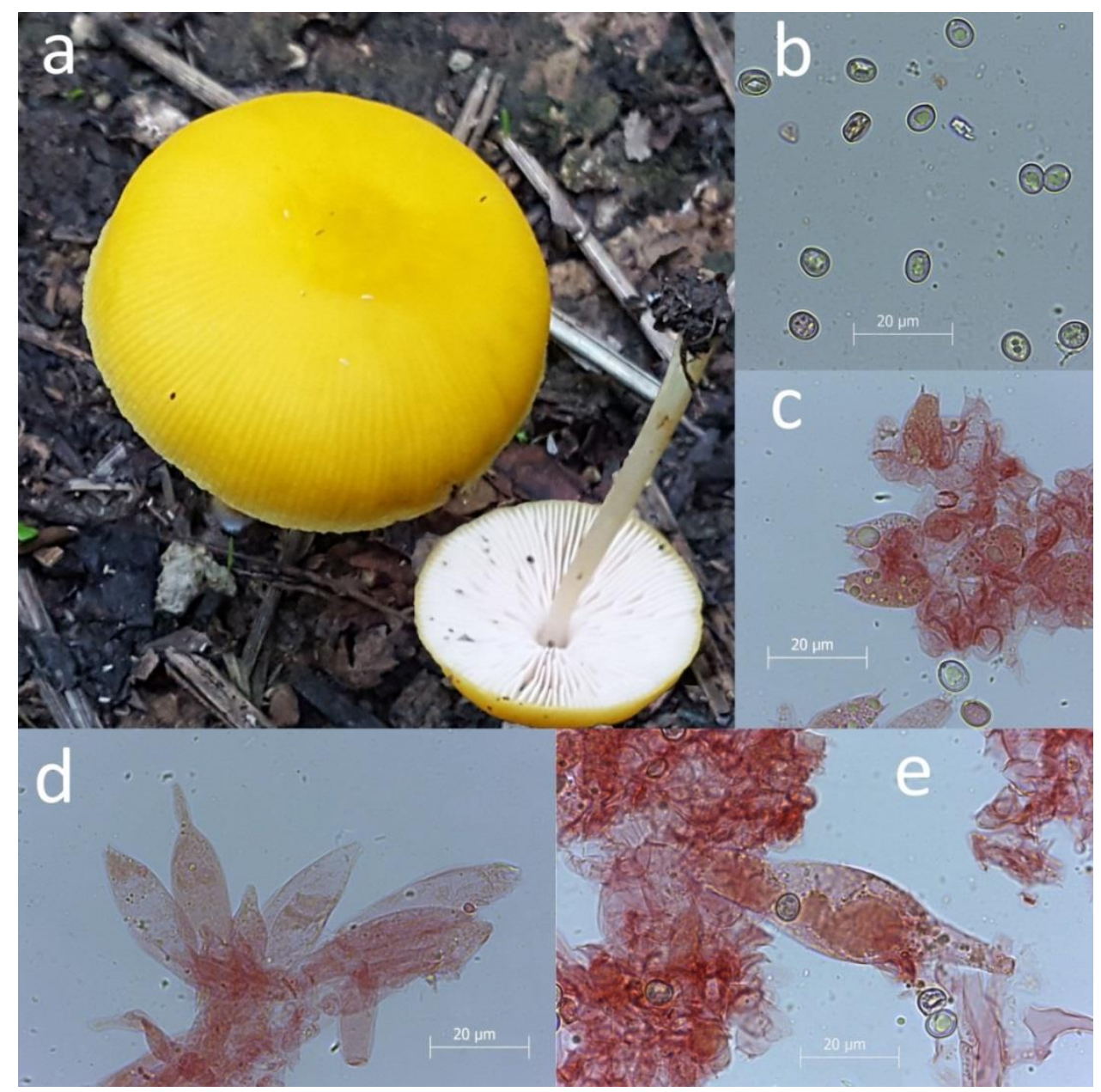

Figure 2. Pluteus variabilicolor a) Basidiocarp b) spores c) basidia d) Cheilocystidia e) Pleurocystidia

\section{Specimen examined}

Turkey - Trabzon, Of, Ballica neighborhood, under Corylus sp. trees, $40^{\circ} 52^{\prime} 845^{\prime \prime} \mathrm{N}$, 40¹6'849"E, 86 m. 27.08.2015, AK. 2963, Genbank number MH 724931. 


\section{Coprinopsis urticicola (Berk. \& Broome) Redhead, Vilgalys \& Moncalvo}

Syn: Agaricus urticicola Berk. \& Broome, Coprinopsis urticicola var. hawaiiensis Keirle, Hemmes \& Desjardin, C. urticicola var. salicicola (Uljé \& Noordel.) Walleyn, Verbeken, Kerckh., Keersm., Christiaens, Esprit, Leyman \& Van de Kerckh., $C$. urticicola var. salicicola (Uljé \& Noordel.) Noordel., C. urticicola (Berk. \& Broome) Redhead, Vilgalys \& Moncalvo, Coprinus brassicae Peck, C. urticicola (Berk. \& Broome) Buller, C. urticicola var. salicicola Uljé \& Noordel., C. urticicola var. urticicola (Berk. \& Broome) Buller, Pilosace urticicola (Berk. \& Broome) Kuntze, Psathyra urticicola (Berk. \& Broome) Sacc.

\section{Macroscopic and microscopic features}

Pileus 0.4-0.8 $\times 0.2-0.4 \mathrm{~cm}$, ellipsoid when young, then ellipsoid or egg-shaped, pure white. Flesh broken up into small white. Lamellae free, initially white, then greyishbrown and eventually black. Stipe 2-4 x 0.5-0.1 cm, cylindrical, surface smooth, white, slightly clavate base. Basidia 17-26 × 6-9.5 $\mu \mathrm{m}$, 4-spored. Spores 5.5-8.7 × 4.7-6.2 $\mu \mathrm{m}$, ellipsoid, pale brown, usually carries a wide germ pore on the base. Cheilocystidia 40$70 \times 12-18 \mu \mathrm{m}$, globose and ellipsoid. Pleurocystidia 45-80 $\times 17-30 \mu \mathrm{m}$, cylindrical, narrowly conical (Figure 3).

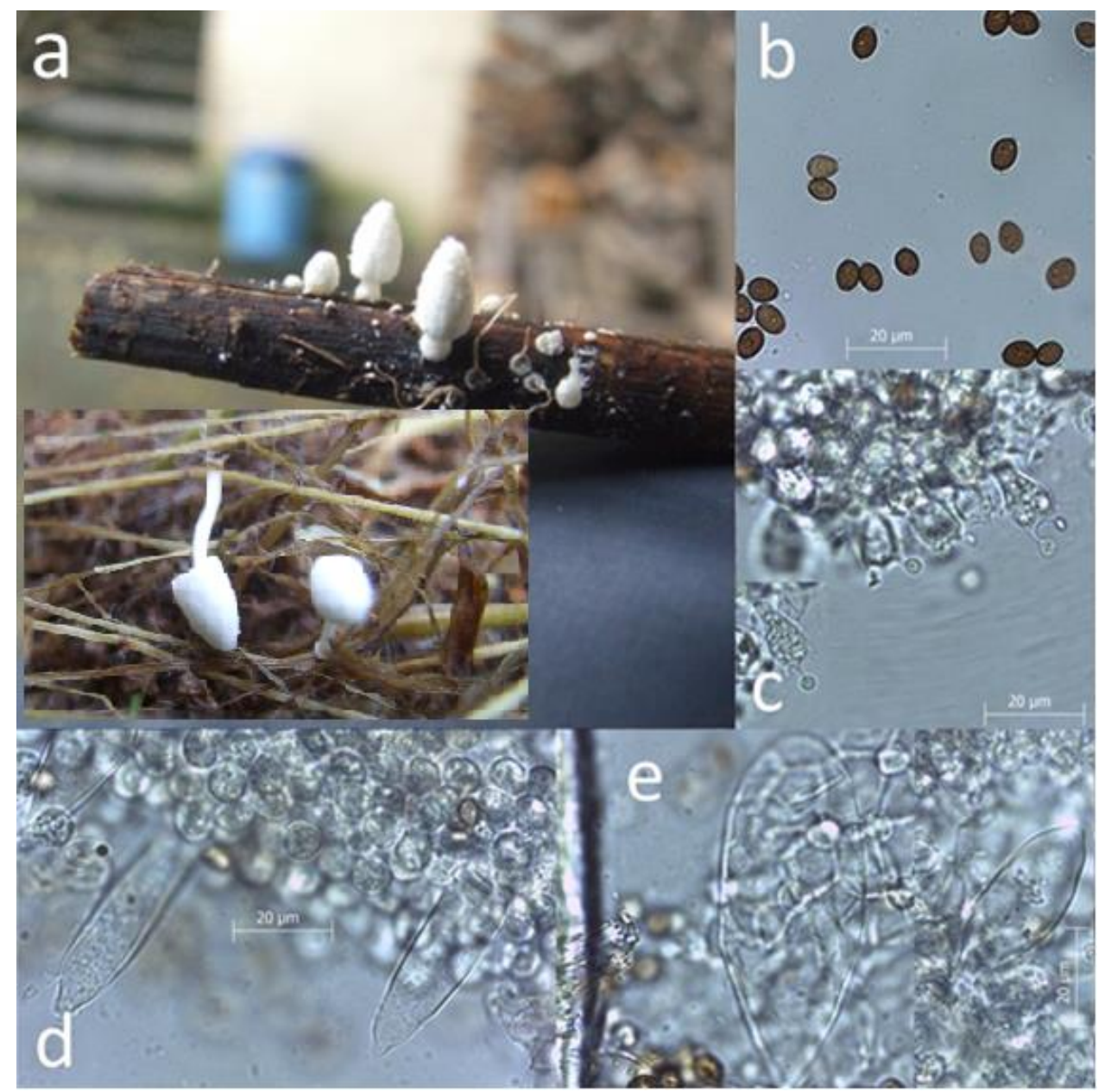

Figure 3. Coprinopsis urticicola a) Basidiocarp b) spores c) basidia d) Cheilocystidia e) Pleurocystidia 


\section{Specimen examined}

Trabzon, Of, Ballica neighborhood, on Urtica sp. remains, 40 $52^{\prime} 492 " \mathrm{~N}$, 40¹6'474"E, 121 m 31.07.2012, AK. 2044, Genbank number MH 748639.

\section{Chroogomphus confusus Y.C. Li \& Zhu L. Yang}

\section{Macroscopic and microscopic features}

Pileus 4-8 $\mathrm{cm}$ wide, convex, surface smooth and bright, hairless, grayish brown or reddish brown. Lamellae decurrent, greyish orange. Stipe 5-10 $\times 1-2 \mathrm{~cm}$, subcylindric, yellowish to pale orange. Basidia 38-60 × 9-18 $\mu \mathrm{m}$, 4-spored. Spores 14-21.6 × 6-7.5 $\mu \mathrm{m}$, smooth, ellipsoid, dextrinoid and dark gray. Cystidia 110-160 × 18-22 $\mu \mathrm{m}$, subclavate (Figure 4).

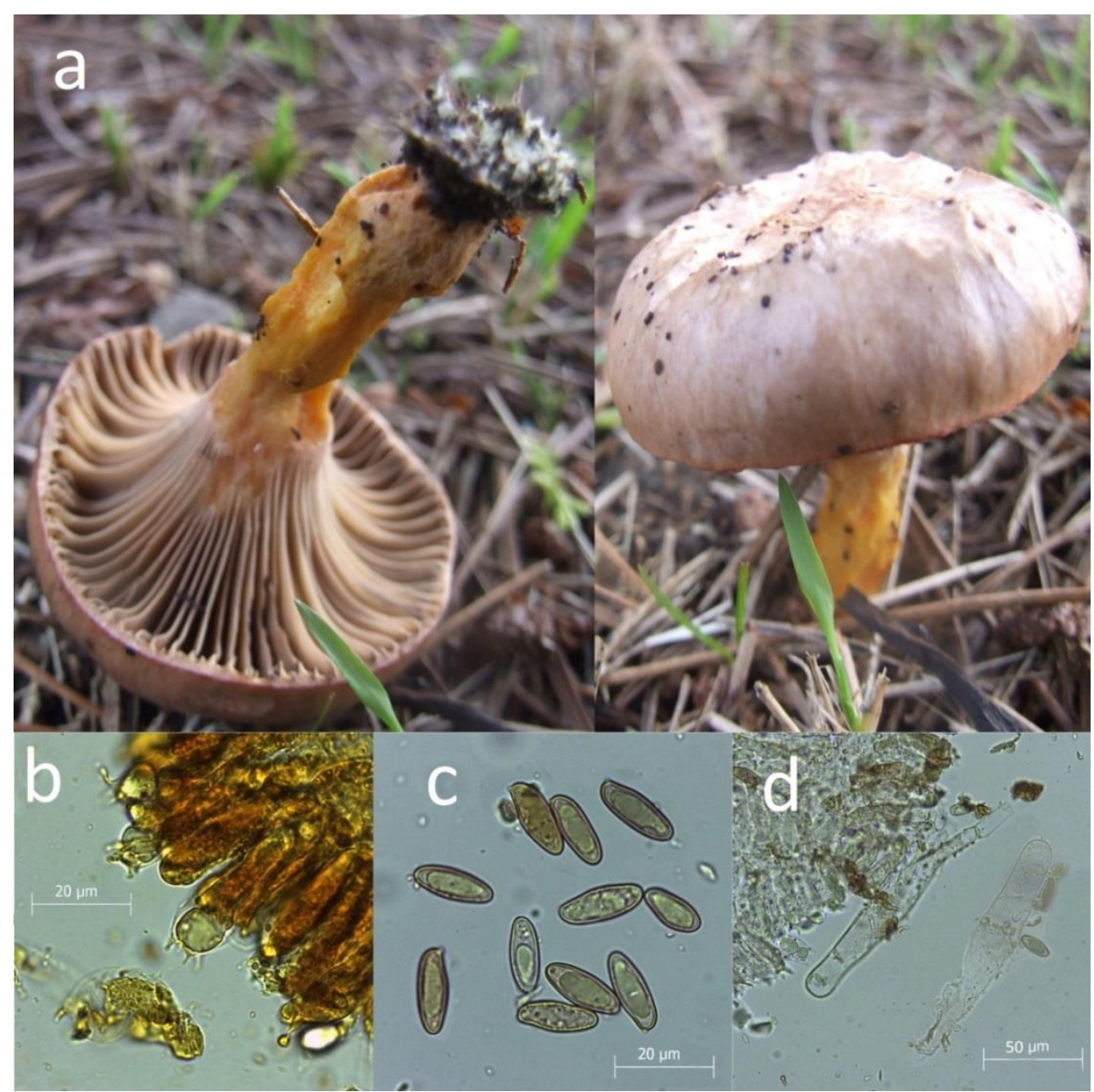

Figure 4. Chroogomphus confusus a) Basidiocarp b) spores c) basidia d) Cheilocystidia

\section{Specimen examined}

Turkey - Trabzon, Çamburnu, around Maritime Faculty, under pine trees, 4055'390"N, 40¹2'596"E, 16m. 29.10.2014, AK. 2871, Genbank number MH 724933. 


\section{Discussion}

With this study three Basidiomycete taxa, Pluteus variabilicolor, Coprinopsis urticicola and Chroogomphus confusus were reported from Turkey for the first time. General characteristics of all the three taxa are in agreement with those given in literature.

Pluteus variabilicolor was erected by Babos (1978) from Hungary. It is generally characterised by yellowish orange pileus colour when young which turns to light yellow later on. It differs from many other Pluteus species by having dimorphic pileipellis and caulocystidial elementsk (Lezzi et al., 2014). Macro and micromorphological characteristics of $\mathrm{P}$. was compared with similar Pluteus species in Table 1.

Table 1. Comparison of Pluteus varibilicolor with similiar Pluteus species (Vizzini and Ercole, 2011; Кио, 2015)

\begin{tabular}{c|c|c|c} 
Species & $\begin{array}{c}\boldsymbol{P} \text {. varibilicolor } \\
\text { Habibat } \text { Corulus } \\
\text { sp. trees }\end{array}$ & $\begin{array}{c}\boldsymbol{P} \text {. aurantiorugosus } \\
\text { on decaying hardwood }\end{array}$ & on decaying hardwood \\
\hline Pileus & $3-6 \mathrm{~cm}$ & $2-5 \mathrm{~cm}$ & $3-5 \mathrm{~cm}$ \\
\hline Pileus color & lemon yellowish & $\begin{array}{c}\text { bright scarlet to orange when young, } \\
\text { fading to orangish yellow in age }\end{array}$ & $\begin{array}{c}\text { Gold-yellow wild } \\
\text { abrown-yellow center }\end{array}$ \\
\hline Stipe & $\begin{array}{c}2.5-6 \times 0.4-0.7 \\
\mathrm{~cm}\end{array}$ & $1.5-4 \times 0.2-0.4 \mathrm{~cm}$ & $4-5(9) \times 0.2-0.5 \mathrm{~cm}$ \\
\hline Basidia & $\begin{array}{c}20-28 \times 5.8-8.3 \\
\mu \mathrm{m}\end{array}$ & $25-30 \times 6-8 \mu \mathrm{m}$ & $20-37 \times 7.5-10 \mu \mathrm{m}$ \\
\hline Spores & $\begin{array}{c}5.4-7 \times 4.5- \\
5.6 \mu \mathrm{m}\end{array}$ & $5.5-8 \times 4-5 \mu \mathrm{m}$ & $5.5-7 \times 5-6 \mu \mathrm{m}$ \\
\hline Cheilocystidia & $\begin{array}{c}40-48 \times 12-15 \\
\mu \mathrm{m}\end{array}$ & $35-45 \times 12.5-22.5 \mu \mathrm{m}$ & $65 \times 12 \mu \mathrm{m}$ \\
\hline Pleurocystidia & $\begin{array}{c}65-120 \times 19-35 \\
\mu \mathrm{m}\end{array}$ & $40-55(60) \times 20-23 \mu \mathrm{m}$ & $100 \times 28 \mu \mathrm{m}$ \\
\hline
\end{tabular}

Coprinopsis urticicola is characterized by its habitat on rotting stems of herbs as well as on fine branchlets, the small fruiting bodies, the branched velar hyphae, and the primarily elliptical spores $<9 \mu \mathrm{m}$ long (Breitenbach and Kränzlin, 1991). Coprinopsis urticicola was compared with morphologically similar species in the following Table 2.

Table 2. Comparison of Coprinopsis urticicola with closer Coprinopsis species (Gierczyk et al., 2011; Amandeep et al., 2012)

\begin{tabular}{c|c|c|c} 
Species & C. urticicola & C. vermiculifer & C. gonophylla \\
Habibat & on herbs remnants & $\begin{array}{c}\text { on burnt places or bare, clayey } \\
\text { soil }\end{array}$ & $\begin{array}{c}\text { on the sandy and stony } \\
\text { meadow }\end{array}$ \\
\hline Pileus size & $\begin{array}{c}0.4-0.8 \times 0.2-0.4 \\
\mathrm{~cm}\end{array}$ & $1.4-1.7 \mathrm{~cm}$ & up to $0.3 \mathrm{~cm}$ \\
\hline Pileus color & pure white & $\begin{array}{c}\text { brownish gray, pileal scales } \\
\text { grayish white }\end{array}$ & $\begin{array}{c}\text { Veil white, breaking into } \\
\text { patches }\end{array}$ \\
\hline Stipe & $2-4 \times 0.5-0.1 \mathrm{~cm}$ & $2.5-2.8 \mathrm{~cm}$ & $6 \times 0.1-0.3 \mathrm{~cm}$ \\
\hline Basidia & $17-27 \times 5.5-10 \mu \mathrm{m}$ & $17-22 \times 12-13.6 \mu \mathrm{m}$ & $15-30 \times 7-9 \mu \mathrm{m}$ \\
\hline Spores & $5.6-8.7 \times 4.7-6.2$ & $(9.3) 10-13.6 \times(6.8) 7.6-9.3 \mu \mathrm{m}$ & $\begin{array}{c}7.0-8.5 \times 6.5-8.0 \times 5.5-6.0 \\
\mu \mathrm{m}\end{array}$ \\
\hline Cheilocystidia & $40-70 \times 12-18 \mu \mathrm{m}$ & $39-59.5 \times 27-35.7 \mu \mathrm{m}$ & $40-85 \times 25-45 \mu \mathrm{m}$ \\
\hline Pleurocystidia & $45-80 \times 17-30 \mu \mathrm{m}$ & $34-85 \times 25.4-37.4 \mu \mathrm{m}$ & $50-120 \times 40-20 \mu \mathrm{m}$ \\
\hline
\end{tabular}


Chroogomphus confusus has a brownish orange to orange pileus, a nonamyloid pileipellis and reduced amyloidity in the pileal trama (Li et al., 2009). Chroogomphus confusus was compared with morphologically similar species in the following Table 3.

Table 3. Comparison of Chroogomhus confusus with closer Chroogomphus species (Li et al., 2009; Martin et al., 2016; Razaq et al., 2016)

\begin{tabular}{c|c|c|c}
\hline Species & C. confusus & \multicolumn{2}{c}{ C. mediterraneus } \\
Habibat & under pine trees & growing under Pinus halepensis & C. rutilus \\
\hline Pileus size & $4-8 \mathrm{~cm}$ & $2.5-7(8) \mathrm{cm}$ & $2-8(10) \mathrm{cm}$ \\
\hline Pileus color & $\begin{array}{c}\text { grayish brown or } \\
\text { reddish brown }\end{array}$ & $\begin{array}{c}\text { grayish, cream-orange, vinaceous } \\
\text { to dingy vinaceous brown }\end{array}$ & $\begin{array}{c}\text { grayish, vinaceous to } \\
\text { dingy vinaceous brown }\end{array}$ \\
\hline Stipe & $5-10 \times 1-2 \mathrm{~cm}$ & $6-10 \times 1-2 \mathrm{~cm}$ & $5-8(12) \times 0.5-1.5 \mathrm{~cm}$ \\
\hline Basidia & $38-60 \times 9-18 \mu \mathrm{m}$ & $50-60 \times 10-15 \mu \mathrm{m}$ & $40-50 \times 12-14 \mu \mathrm{m}$ \\
\hline Spores & $\begin{array}{c}14-21.6 \times 6-7.5 \\
\mu \mathrm{m}\end{array}$ & $\begin{array}{c}(15.5) 16-19(21) \times(5.5) 6-7.5(8) \\
\mu \mathrm{m}\end{array}$ & $\begin{array}{c}16.5-19(21) \times(5.5) 6-8 \\
\mu \mathrm{m}\end{array}$ \\
\hline Cheilocystidia & $\begin{array}{c}110-160 \times 18-22 \\
\mu \mathrm{m}\end{array}$ & $(90) 100-175 \times 17-21 \mu \mathrm{m}$ & $118-170 \times 16-23 \mu \mathrm{m}$ \\
\hline Pleurocystidia & & $(90) 100-175 \times 17-21 \mu \mathrm{m}$ & $118-170 \times 16-23 \mu \mathrm{m}$ \\
\hline
\end{tabular}

\section{Conclusion}

Pluteus variabilicolor, Coprinopsis urticicola and Chroogomphus confusus were given as new records from Turkey, increasing the current taxa numbers of the genera Pluteus, Coprinopsis and Chroogomphus, in Turkey, to 24, 25 and 2 respectively. The current determined taxa number and the current plant biodiversity of Turkey indicate that, macrofungal biodiversity studies should be handled with top priority.

\section{REFERENCES}

[1] Akata, I., Uzun, Y., Kaya, A. (2016): Macrofungial diversity of Zigana Mountain. Biological Diversity and Conservation 9(2): 57-69.

[2] Allı, H., Çöl, B., Şen, İ. (2017): Macrofungi biodiversity of Kütahya (Turkey) province. Biological Diversity and Conservation 10(1): 133-143.

[3] Amandeep, K., Atri, N. S., Munruchi, K. (2014): Taxonomic study on coprophilous species of Coprinopsis (Psathyrellaceae, Agaricales) from Punjab, India. - Mycosphere 5(1): 1-25.

[4] Babos, M. (1978): Pluteus studies, I. (Basidiomycetes, Pluteaceae). - Annales HistoricoNaturales Musei Nationalis Hungarici, Budapest 70: 93-97.

[5] Breitenbach, J., Kränzlin, F. (1991): Fungi of Switzerland. - Volumes 4. Verlag Mykologia, Lucerne, Switzerland.

[6] Desjardin, D. E., Wood, M. G., Stevens, F. A. (2015): California Mushrooms: The Comprehensive Identification Guide. - Timber Press: Portland, OR. 560 p. USA.

[7] Dündar, Ö., Demircioğlu, H., Özkaya, O., Dündar, B. (2016): Kültür mantarlarının muhafazası ve kalite özellikleri üzerine yapılan araştırmalar. - Turkish Journal of Agriculture: Food Science And Technology 4(3): 150-154.

[8] Gardes, M., Bruns, T. D. (1993): ITS primers with enhanced specificity for basidiomycetes-application to the identification of mycorrhizae and rusts. - Molecular Ecology 2: 113-118.

[9] Gierczyk, B., Kujawa, A., Pachlewski, T., Szczepkowski, A., Wójtowski, M. (2011): Rare species of the genus Coprinus Pers. s. Lato. - Acta Mycol 46(1): 27-73. 
[10] Gierczyk, B., Kujawa, A., Szczepkowski, A. (2014): New to Poland species of the broadly defined genus Coprinus (Basidiomycota, Agaricomycotina. - Acta Mycol 49(2): 159-188.

[11] Iş1k, H., Türkekul, İ. (2017): A new record for Turkish mycota from Akdağmadeni (Yozgat) province: Russula decolorans (Fr.) Fr. - Anatolian Journal of Botany 1: 1-3.

[12] Kaya, A. (2015): Contributions to the Macrofungal Diversity of Atatürk Dam Lake Basin. - Turkish Journal of Botany 39: 162-172.

[13] Kaya, A., Uzun, Y. (2018): New Contributions to the Turkish Ascomycota. - Turkish Journal of Botany 42: 644-652.

[14] Kaya, A., Uzun, Y., Karacan, İ. H., Yakar, S. (2016): Contributions to Turkish Pyronemataceae from Gaziantep province. - Turkish Journal of Botany 40: 298-307.

[15] Keleş, A., Oruç, Y. (2017): Leucocoprinus brebissonii (Godey) Locq, A New Record for Turkish Mycobiota. - Anatolian Journal of Botany 1(2): 49-51.

[16] Kirk, P. F., Cannon, P. F, Minter, D. W., Stalpers, J. A. (2008): Dictionary of the Fungi. 10th ed. Wallingford, UK: CAB International.

[17] Kuo, M. (2015): Pluteus aurantiorugosus. - MushroomExpert.Com http:// www.mushroomexpert.com/pluteus_aurantiorugosus.html.

[18] Lezzi, T., Vizzini, A., Ercole, E., Migliozzi, V., Justo, A. (2014): Phylogenetic and morphological comparison of Pluteus variabilicolor and P. castri (Basidiomycota, Agaricales). - Ima Fungus 5(2): 415-423.

[19] Li, Y. C., Yang, Z. L., Tolgor, B. (2009): Phylogenetic and biogeographic relationships of Chroogomphus species as inferred from molecular and morphological data. - Fungal Diversity 38: 85-104.

[20] Martin, M. P., Siquier, J. L. I., Salom, J. C., Telleria, M. T., Finschow, G. (2016): Barcoding sequences clearly separate Chroogomphus mediterraneus (Gomphidiaceae, Boletales) from C. rutilus, and allied species. - Mycoscience 57(6): 384-392.

[21] Migliozzi, V. (2011): Pluteus variabilicolor, specie frequente nella cerreta di Macchiagrande di Manziana (RM). - Parliamo di Funghi 19(1): 3-9.

[22] Razaq, A., Ilyas, S., Khalid, A. N. (2016): Molecular Identification of Chinese Chroogomphus roseolus from Pakistani Forests, a Mycorrhizal Fungus, Using ITS-rDNA Marker. - Pak. J. Agri. Sci. 53(2): 393-398.

[23] Sadullahoğlu, C., Demirel, K. (2018): Flammulina fennae Bas, A new record from Karz Mountain (Bitlis). - Anatolian Journal of Botany 2(1): 19-21.

[24] Sesli, E. (2018): Cortinarius ve Lyophyllum Cinslerine Ait Yeni Kayıtlar. - Mantar Dergisi 9(1): 18-23.

[25] Sesli, E., Denchev, C. M. (2014): Checklists of the myxomycetes, larger ascomycetes, and larger basidiomycetes in Turkey. - 6th edn. Mycotaxon, Checklists Online (http://www.mycotaxon.com/resources/checklists/sesli-v106-checklist.pdf): 1-136.

[26] Solak, M. H., Işıloğlu, M., Kalmış, E., Allı, H. (2015): Macrofungi of Turkey, Checklist, Volume II. - Üniversiteliler Ofset, Bornova, İzmir.

[27] Uzun, Y., Acar, İ. (2018): A New Inocybe (Fr.) Fr. Record for Turkish Macrofungi. Anatolian Journal of Botany 2(1): 10-12.

[28] Uzun, Y., Kaya, A. (2018): Leucocoprinus cepistipes, A New Coprinoid Species Record for Turkish Macromycota. - Süleyman Demirel University, Journal of Natural and Applied Sciences 22(1): 60-63.

[29] Uzun, Y., Acar, İ., Akçay, M. E., Kaya, A. (2017): Contributions to the macrofungi of Bingöl, Turkey. - Turkish Journal of Botany 41: 516-534.

[30] Uzun, Y., Karacan, İ. H., Yakar, S., Kaya, A. (2018): New additions to Turkish Tricholomataceae. - Anatolian Journal of Botany 2(2): 65-69.

[31] Vizzini, A., Ercole, E. (2011): A new annulate Pluteus variety from Italy. - Mycologia, 103(4): 904-911. 
[32] Webster, J., Weber, R. (2007): The Fungi. - Third Edition. Cambridge University Press, The Edinburgh Building, Cambridge CB2 8RU, UK.

[33] White, T. J., Bruns, T., Lee, S., Taylor, J. (1990): Amplification and direct sequencing of fungal ribosomal RNA genes for phylogenetics. - In: Innis, M. A., Gelfand, D. H., Shinsky, J. J., White, T. J. (eds.) A Guide to Methods and Applications. Academic Press. 